\title{
Stratigraphische Untersuchungen an molluskenführenden Terrassensedimenten und ihren Deckschichten im mittleren Neckarbecken (Württemberg)
}

\author{
Erhard Bibus \& Wolfgang RäHle *)
}

Keywords: Quartär, Mittelpleistozän, Jungpleistozän, Flussterrassen, Hochflutlehme, Schotter, Terrassen, Paläoböden, Molluskenfauna, Geomorphologie, Neckargebiet, Baden-Württemberg,

TK 25: 6721, 7020

Zusammenfassung: Im Rahmen geomorphologischer Untersuchungen von Flussterrassen und ihren Deckschichten werden im mittleren Neckarraum drei neue Aufschlüsse beschrieben und die darin enthaltenen fossilen Molluskenfaunen vorgestellt. In zwei Fällen (Heilbronn-Böckingen und Obereisesheim) handelt es sich bei den fossilführenden Sedimenten um Sande, Kiese und Hochflutlehme, welche die vorletztkaltzeitlichen Schotter der 5-7 m-Terrasse i.S.v. Bibus (2002) abschließen. Die Molluskenfunde bestätigen, dass es sich im mittleren Neckarbecken bei Hochflutsedimenten auf der 5-7 m-Terrasse um warmzeitliche Bildungen handelt, welche in das Eem gestellt werden müssen. Die gleiche Höhenlage der Vorkommen belegt, dass es nach dem Eem im Heilbronner Raum zu keiner großräumigen Tektonik gekommen ist.

In Bietigheim-Bissingen wurden Mittelterrassenschotter (Basis ca. 20 m über Enzniveau) sowie deren Deckschichten untersucht. Die überaus artenreiche Molluskenfauna mit Theodoxus serratiliniformis, Cochlostoma scalarinum saueri u.a. beweist, dass es sich dabei um ein Äquivalent derjenigen Schotter handelt, deren Molluskenfauna bereits 1914 von

*Anschrift der Verfasser:

Prof. Dr. E. Bıbus, Geographisches Institut der Universität Tübingen, Hölderlinstraße 12, D-72074 Tübingen; Dr. W. RäHle, Zoologisches Institut der Universität Tübingen, Auf der Morgenstelle 28, D72076 Tübingen
GEYER veröffentlicht wurde, und die seitdem zu den bedeutendsten Fundstellen mittelpleistozäner Mollusken in Deutschland zählt. Aufgrund der terrassenmorphologischen Situation sowie zusätzlicher Nachweise chronostratigraphisch aussagekräftiger Molluskenarten, war es erstmals möglich, eine genauere Altersbestimmung durchzuführen. Alle bisher vorliegenden Daten sprechen dafür, dass es sich bei den molluskenführenden Mittelterrassenschottern von Bietigheim-Bissingen um Bildungen der fünftletzten Warmzeit (OIS 13, Cromer IV i.S.v. ZAGWIJN 1989) handelt.

Abstract: In the context of geomorphologic studies, results from three new river terrace exposures in the middle Neckar region (SW-Germany) are presented. At two sites (Heilbronn-Böckingen and Obereisesheim) mollusc bearing sediments of sand, gravel, and flood plain loam overlay the gravels of the 5-7 meter terrace sensu Bibus (2002). The fossil record confirms that the flood plain sediments on the 57 meter terrace origin from a warm period, which must be the Eemian. Since these sediments occur at the same altitude it is concluded that there have been no wide spread tectonic processes in the Heilbronn region after the Eemian.

Middle terrace-gravels (base about $20 \mathrm{~m}$ above the level of present river Enz) and their respective cover layers were investigated at the site Bietigheim-Bissingen. The species rich mollusc fauna with Theodoxus serratiliniformis, Cochlostoma scalarinum saueri proves that these deposits are an equivalent of the gravels investigated by GEYER in 1914. Since this publication, the same fauna still belongs to the most important findings of middle Pleistocene molluscs in Germany. Based on the terrace-morphologic setting and supported by evidence from chronostratigraphi- 
cally significant mollusc species, it was possible to determine the age more precisely. All available data suggest that the mollusc-bearing middle terrace-gravels origin from the fifth last warm period (OIS 13, Cromer IV sensu ZAGWIJN 1989).

\section{Einleitung}

Aufgrund des stark zurückgegangenen Kiesabbaus im mittleren Neckarraum wurden in jüngerer Vergangenheit kaum mehr stratigraphisch bedeutungsvolle Fossilien in den Ablagerungen des Neckars und seiner Nebenflüsse gefunden. Vereinzelt lassen sich jedoch in den Terrassensedimenten Schnecken nachweisen, die manchmal Alterseinstufungen oder zumindest allgemeine Klimaaussagen zulassen.

Von den Autoren wurden in früheren Arbeiten Fundstellen bei Rottenburg und Heilbronn behandelt (Bibus \& RÄhle 1986, Rähle \& Bibus 1992). Nunmehr sind weitere Fundpunkte in unterschiedlich hoch über der Talaue gelegenen Schotterkörpern bzw. deren abdeckenden Hochflutlehmen hinzugekommen. Es handelt sich um Höhenschotter im Eyachtal bei Haigerloch und im Neckartal bei Rottenburg (vgl. Bibus \& RÄhle 2003) sowie verschiedene Funde im so genannten „Hochterrassenbereich“ im mittleren Neckarbecken. In dieser Arbeit soll über ein Mittelterrassenvorkommen der Enz bei Bietigheim-Bissingen und mehrere, nur wenige Meter über der Talaue gelegene Terrassenkörper des Neckars im Heilbronner Becken berichtet werden (vgl. Abb. 1).

\section{Die cromerzeitlichen Mittelterrassenschotter von Bietigheim-Bissingen im Enztal}

Im unteren Enztal ist über dem nördlichen Talhang gegenüber der Ortschaft Bissingen seit längerer Zeit in den Deckschichten des Muschelkalkbruchs Fink ein Schotter aufge- schlossen, der nach durchgeführten Vermessungen mit seiner Basis 20,16 m über dem Enzniveau in 201, $6 \mathrm{~m}$ ü.NN dem Muschelkalk auflagert. Die Schotteruntergrenze weist flache Mulden auf, so dass die Auflagerungshöhe um ca. 1 m schwanken kann. Mit einem Höhenmesser konnte die Schotteruntergrenze in ca. $19 \mathrm{~m}$ über der Aue eingemessen werden. Die Schotter treten zur Talkante und im randlichen Bereich der Terrassenverebnung z.T. an die Oberfläche, in weiten Bereichen werden sie von unterschiedlich stark gegliederten und in der Mächtigkeit schwankenden Lößdeckschichten überlagert. Von Bedeutung ist die Tatsache, dass auch die Mächtigkeit der Enzschotter mit geringer werdenden bzw. fehlenden Deckschichten abnimmt. Dies ist dadurch zu erklären, dass mit geringer werdenden und einfacher gegliederten Deckschichten die Schotter tiefer entkalkt wurden und sie dadurch einen beachtlichen Volumenverlust erfahren haben. Die Ursache für die zunehmende Entkalkung und die geringe Schottermächtigkeit liegt somit in den längeren, zur Verfügung stehenden Verwitterungszeiträumen bei nur zeitweiligem oder insgesamt fehlendem Schutz durch abdeckende Lößschichten. Darüber hinaus kann der Schotterkörper zur Terrassenkante hin auch teilweise erodiert sein.

In der NW-Ecke des Aufschlusses werden die Deckschichten bis zu $6 \mathrm{~m}$ mächtig und sind durch eine Humuszone und drei pseudovergleyte fossile Bt-Horizonte gegliedert. Die Schotter erreichen an dieser Stelle eine maximale Mächtigkeit von ca. $6 \mathrm{~m}$ und sind unverwittert. In den Schotterkörper ist eine 2 m tiefe Erosionsstufe eingeschnitten (vgl. BiBus 2002: 141f.).

Zur Terrassenkante hin reduzieren sich die Schotter auf eine Mächtigkeit von ca. $3 \mathrm{~m}$. Sie sind hier verlehmt und weitgehend entkalkt. Nur im untersten Bereich können unmittelbar über dem Muschelkalk noch einzelne größere Muschelkalkgerölle auftreten. Die Schotter weisen eine wirre Lagerung auf, was auf das Weglösen der Kalkgerölle und damit verbun- 


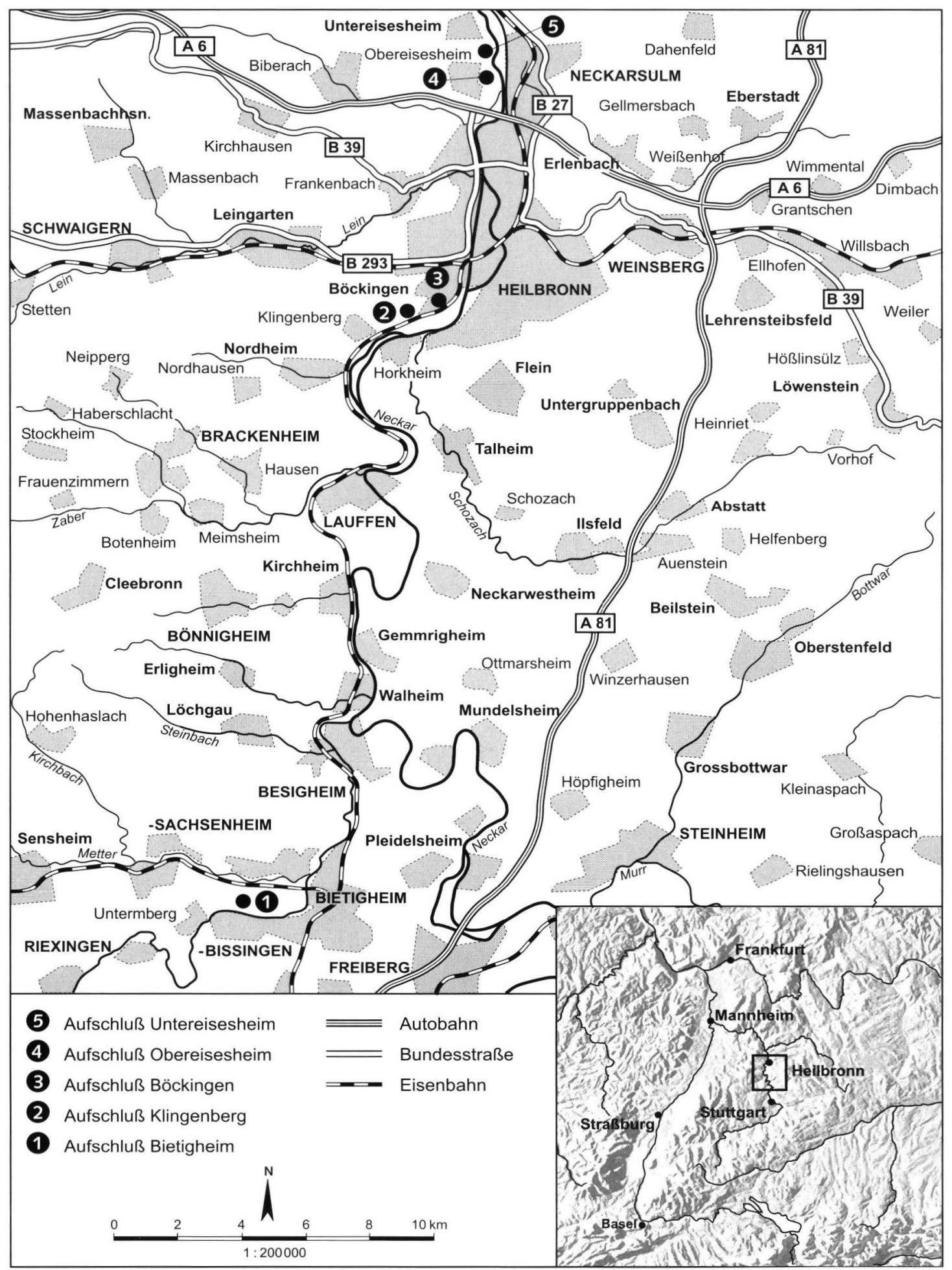

Abb. 1: Übersichtskarten mit Lage der im Text behandelten Profile

Fig. 1: General map showing the location of the records mentioned in the text. 
dene Sackungen zurückzuführen ist. Durchgeführte Schotteranalysen aus frischem Kies haben im Grobkies 53,5\% und im Mittelkies $54,5 \%$ Kalkgerölle erbracht, so dass bei der Verwitterung des Schotterkörpers über $50 \%$ der Gerölle in den angegebenen Fraktionen weggelöst wurden.

Für die Stratigraphie ist von Bedeutung, dass an einer Stelle im mittleren Bereich des Aufschlusses (TK 25:Bl. 7020 Bietigheim-Bissingen; ca. R 350821 H 542365) innerhalb des Schotterkörpers Schneckenfunde gemacht werden konnten. Bereits Geyer (1914) und später WARTH (mündl. Mitt.) haben in diesem Schotterzug Mollusken beobachtet. Bei unseren Aufnahmen war der Schotterkörper an der Fundstelle von einer 1,40 m mächtigen Lößdeckschicht überlagert, an deren Basis verlagertes Bodenmaterial auftrat (vgl. Abb. 2). Der Schotterkörper setzte mit einem $50 \mathrm{~cm}$ mächtigen, sandig-lehmigen Kies ein, der in einen lehmigen, vereinzelt Kiese führenden Sand überging. Die beschriebenen Schichten waren entkalkt und von einem Bvt-Horizont überprägt. Nach unten folgte eine flache Rinnenfüllung aus himbeerfarbenen Grobsanden mit einzelnen Kiesen und fahlrötlichen Lehmschmitzen. In den kalkhaltigen Sedimenten waren häufig Schnecken eingelagert. Darunter trat ein über $1 \mathrm{~m}$ mächtiger, kalkhaltiger Kies und ein $10 \mathrm{~cm}$ mächtiges, olivgraues, sandigschluffiges Lehmband auf, in dem auch vereinzelt Schnecken nachgewiesen werden konnten. Nach unten wurde der Schotterkörper von einem ca. 1,40 m mächtigen, sandigen Kies abgeschlossen, dessen Gerölle mit zunehmender Tiefe gröber wurden und der nahe der Basis grobe Blöcke aus Buntsandstein und Muschelkalk führte. Insgesamt wies deshalb der untere Bereich des Sediments ein Erscheinungsbild auf, wie es für periglaziale Schotter typisch ist. Für die darüber eingeschaltete sandige Rinnenfüllung haben sich hingegen aufgrund der Molluskenführung interglaziale Klimaverhältnisse ergeben.
Die Enzschotter von Bietigheim-Bissingen gehören zu den bedeutendsten deutschen Fundstellen, an denen Mollusken aus einer mittelpleistozänen Warmzeit überliefert sind. Geyer (1914) hat diese Vorkommen als Erster entdeckt und bearbeitet. Die betreffenden Schotter befanden sich 20 bis $22,5 \mathrm{~m}$ über dem Enzniveau und waren seinerzeit in zwei Kiesgruben am Weg zwischen Bietigheim und Bissingen aufgeschlossen. GEYER konnte in sandigen Lagen zwischen den Schottern nicht weniger als 82 verschiedene Molluskenarten identifizieren und beschrieb gleichzeitig zwei für die Wissenschaft neue Taxa: Theodoxus serratiliniformis und Cochlostoma scalarinum saueri. In der Folgezeit waren die Aufschlüsse offenbar nicht mehr zugänglich.

Im Jahr 1990 entdeckten unabhängig voneinander Dr. M. Warth (Staatliches Museum für Naturkunde in Stuttgart) und E. Bibus im Steinbruch Fink, an der Straße von Bietigheim nach Untermberg, unmittelbar über dem Oberen Muschelkalk einen teilweise fossilführenden Schotterkörper in einem den GEYER'schen Fundstellen entsprechenden Niveau über der Enz. Auch hier erwiesen sich sandige Lagen als überaus reich an Mollusken. Herr Dr. Warth hat Sedimentproben entnommen und uns für die Bearbeitung der Mollusken zur Verfügung gestellt, wofür wir uns herzlich bedanken möchten. Außerdem wurde von E. Bibus eine Probe in der oben beschriebenen Abfolge geborgen (vgl. Abb. 2). Die Fundumstände und - wie sich bald herausstellte - die weitgehende Übereinstimmung in der Zusammensetzung der Molluskenfauna, sprechen eindeutig dafür, dass es sich bei den Schottern aus dem Steinbruch Fink und den Schottern, welche GEYER untersucht hat, um äquivalente Bildungen handelt.

Die Geyerschen Molluskenfunde belegen lediglich ein mittelpleistozänes Alter der Schotter. Eine genauere Altersbestimmung war bisher nicht möglich (Mejjer 1988). Ergebnisse neuerer Untersuchungen über die 


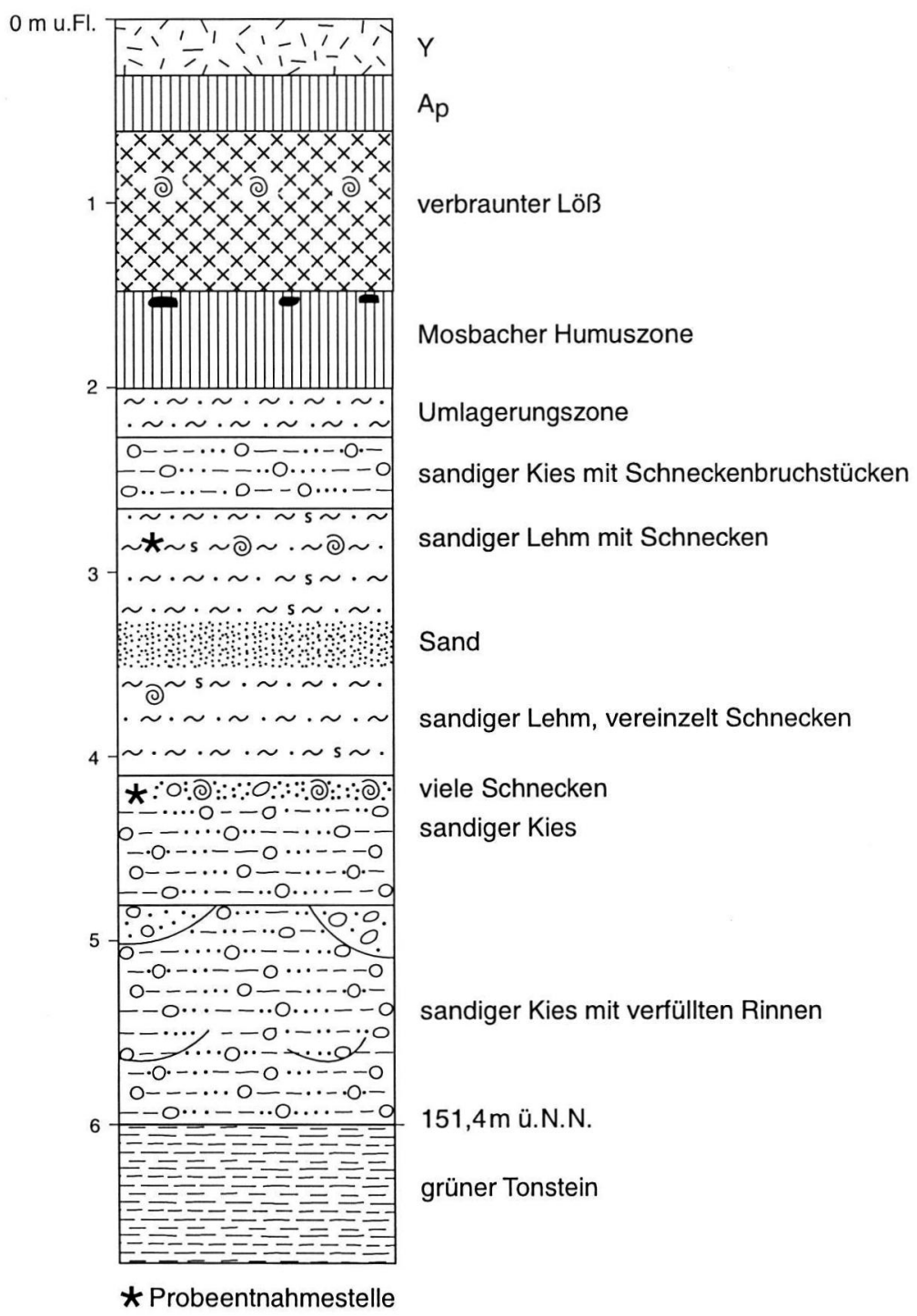

Abb. 2: Profilausschnitt mit den schneckenführenden Rinnensedimenten im Kalksteinbruch Fink in Bietigheim-Bisingen

Fig. 2: Section of the recordshowing the snail bearing sediments in the lime quarry Fink at BietigheimBissingen

Flussterrassen und ihre Deckschichten im Untersuchungsraum (Bibus 1989b, Bibus \& WesLER 1995, Bibus 2002) sowie zusätzliche Funde chronostratigraphisch besonders wichtiger und aussagekräftiger Arten lassen nun eine erneute Diskussion der Altersfrage zu.

Der überwiegende Teil der Molluskenfunde aus dem Steinbruch Fink stammt aus der mittleren und unteren Hälfte des Schotterkörpers. Eine ganze Reihe der bei GEYER (1914) aufgeführten Arten war in den vorliegenden Sedimentproben nicht enthalten. Andererseits konnten 20 Arten nachgewiesen werden, die bei Geyer noch nicht verzeichnet waren, so dass jetzt insgesamt 103 Taxa aus den Enzschottern bekannt sind (Tabelle 1). 
Tab. 1: Liste der bisher aus den mittelpleistozänen Enzschottern von Bietigheim-Bissingen bekannten Landund Süßwassermollusken (Geyer 1914, JaeCKel 1962, Bibus \& Rähle 2003). Arten, die nur bei Geyer und JAECKEL erwähnt werden, sind durch ein dahinter gesetztes G bzw. J zu erkennen. Vor den Namen der erstmals nachgewiesenen Arten steht das Zeichen “+”.

Tab. 1: List of the land and freshwater molluscs hitherto known from the Middle Pleistocene gravels of the river Enz at Bietigheim-Bissingen (Geyer 1914, JaEckel 1962, Bibus \& Rähle 2003). Species mentioned only by Geyer and Jaeckel are marked by $\mathrm{G}$ or J behind the name. Species mentioned for the first time are marked with “+”.

\section{LIMNISCHE MOLLUSKEN}

1.Fließgewässer

Theodoxus serratiliniformis (GEYER 1914)

Ancylus fluviatilis O.F.MüLL.ER 1774

Unio crassus PIILIIPsson 1788

Pisidium amnicum (O. F. Mül...ER 1774)

+ Pisidium henslowanum (SHEppard 1825)

Pisidium moitessierianum (PALAdL.н 1866) - J

Pisidium supinum A.ScHм1DT 1851

+ Pisidium tenuilineatum STELFOX 1918

2. Quellen und Quellbäche

Belgrandia germanica CL.essin 1882

Pisidium personatum МАлм 1855

3. Stehende und fließende Gewässer Bithynia tentaculata (LINNAEUS 1758)

+ Bithynia leachii (SIIEPPARD 1823) Valvata piscinalis (O. F. MÚLLER 1774)

Radix ovata (Drapanaud 1801)

+ Radix peregra (O. F. MÜL.LER 1774) Bathyomphalus contortus (LinNAFus 1758)

Gyraulus albus (O. F. Mǘ...er 1774)

Sphaerium corneum (LiNNAEUS 1758)

Pisidium casertanum (Pol. 1791)

+ Pisidium nitidum JENyns 1832

+ Pisidium subtruncatum MaLm 1855

4. Stehende Gewässer

Acroloxus lacustris (LinNAEus 1758)

Lymnaea stagnalis (LINNAEUS 1758) - G

Planorbis carinatus (O. F. MǗ...eR 1774)

Anisus vorticulus (Trosches. 1834)

Gyraulus cf. acronicus (A. FÉRUssAc 1807) - G

Gyraulus laevis (ALDFR 1838) - G

Gyraulus crista (LinNaEus 1758)

Pisidium milium HeL.D 1836

5. Kleingewässer und Sümpfe

Valvata cristata O. F. MǘL..er 1774

Galba truncatula (O. F. MÜLLER 1774)

Stagnicola palustris aggr.

Aplexa hypnorum (LinNaEus 1758)-G

Planorbis planorbis (Linnaeus 1758)

Anisus leucostoma (MILlet 1813)

Segmentina nitida (O. F. MüLLER 1774) - G

\section{TERRESTRISCHE MOLLUSKEN}

1. Ausgesprochene Waldarten

Platyla polita (Hartmann 1840) - G

Azeca goodalli (A. FÉruSSAC 1821)

Acanthinula aculeata (O. F. MÜLler 1774) - G

Vertigo pusilla O. F. MÜL.L.ER 1774

Ena montana (DRAPARNAUD 1801)

Cochlodina laminata (MonTAGu 1803)

Macrogastra attenuata lineolata (HELD 1836)

+ Macrogastra densestriata (Rossm. 1836)

Macrogastra plicatula (Draparnaud 1801)

Macrogastra ventricosa (Draparnaud 1801)

+ Clausilia cruciata (STUDER 1820)

+ Fusulus interruptus (C. PFeIfFer 1828)

Discus ruderatus (A. Férussac 1821)

Discus perspectivus (M. v. MÜILIFELD 1816)

+ ? Zonitoides sepultus Lozek 1964

+ Semilimax semilimax (J. Férussac: 1802)

Vitrea diaphana (StUder 1820) - G

+ Aegopis cf. acieformis (V. KLEIN 1847)

+ Aegopis klemmi Sch Lсккuм \& Lo7.ek 1965

Aegopinella nitens / nitidula

Aegopinella pura (ALDER 1830)

+? Aegopinella ressmanni (WESTERL. 1883)

Helicodonta obvoluta (O. F. MÖ.L.Ler 1774)

Petasina edentula (Draparnaud 1805)

+ Monachoides incarnatus (O. F. MÜLLER 1774)

Isognomostoma isognomostomos (SCHRÖTER 1784)

2. Andere Schatten liebende Arten

Cochlostoma scalarinum saueri (GEYER 1914)

Clausilia cf. bidentata (SтRӧм 1765) - G

Clausilia dubia Draparnaud 1805

Clausilia pumila C. PFeIFFer 1828

Discus rotundatus (O.F.MÚLLER 1774)

Vitrea crystallina (O.F.MüLL.LR 1774)

Fruticicola fruticum (O.F.MǗLLER 1774)

Trichia striolata (C. PFeIFFer 1828)

Trichia villosa (Draparnaud 1805)

Perforatella bidentata (GMeLin 1791)

Arianta arbustorum (LinNaEUS 1758)

Helicigona lapicida (LinNaEUS 1758)

Cepaea hortensis (O. F. MÜLL.ER 1774)

Cepaea nemoralis (LinNaEus 1758) - G

3. Ubiquisten

+ Carychium tridentatum (RIsso 1826) 
Cochlicopa cf.lubrica (O.F.MÜLLER 1774)

Cochlicopa cf.lubricella (RossmässLer 1835)

Vertigo angustior JEFFrEYs 1830

Clausilia rugosa parvula A. FÉRUSSAC 1807

Succinella oblonga (DrAPARNAUD 1801)

Punctum pygmaeum (Draparnaud 1801)

Euconulus fulvus (O. F. MÜLL.er 1774)

Perpolita hammonis (STRöм 1765)

Perpolita petronella (L. Pfeiffer 1853) - G

+ Agriolimacidae/Limacidae (kleine Arten)

Trichia hispida (Linnaeus 1758)

4. Offenlandarten

Pupilla muscorum (Linnaeus 1758)

+ Pupilla triplicata (Studer 1820)

Vallonia costata (O. F. MülLER 1774)

Vallonia excentrica STERKI 1893

Vallonia pulchella (O. F. Múller 1774)

Truncatellina cylindrica (A. FÉRUSSAC. 1807) - G

Vertigo pygmaea (DRAPARNAUD 1801)

Cecilioides acicula (O. F. MÚlLER 1774) - G*

5. Sumpfmollusken

Carychium minimum O. F. MÜL.LER 1774

Vallonia enniensis (GREDI.ER 1856)

Vertigo antivertigo (DRAPARNAUD 1801)

+ Vertigo moulinsiana (Dupuy 1849)

+ Succinea putris (LinNaEus1758)

Oxyloma elegans (Risso 1826)

Zonitoides nitidus (O. F. MUL.LER 1774)

Pseudotrichia rubiginosa (RosSMẌSSLER 1838)

*) Cecilioides acicula lebt unterirdisch. Die Funde lassen sich deshalb nicht eindeutig der vorliegenden Fauna zuordnen.

An dieser Stelle möchten wir uns bei Herrn Dr. M. Adler (Gomaringen), der die Bestimmung der Erbsenmuscheln der Gattung Pisidium übernommen hat, sowie bei Herrn $\mathrm{H}$. Nordsieck (Aarbergen-Rückershausen), welcher die Bestimmung der Schließmundschnecken (Clausiliidae) überprüfte, sehr herzlich bedanken.

Die vorliegende Artenliste (Tabelle 1) gibt eine gute Übersicht über die damalige Molluskenfauna des Enztales. Die Angehörigen der limnischen Komponente, zu der auch Sumpfund Uferbewohner gezählt werden können, ist ausgesprochen dominant. Über $80 \%$ der ausgelesenen Individuen gehören zu dieser Gruppe. Bei den meisten übrigen Arten handelt es sich um Angehörige einer reich differenzierten, feucht liebenden Auwaldfauna. Offenlandarten spielen nur eine sehr untergeordnete Rolle und werden vor allem von Arten repräsentiert, die auch auf versumpften Flächen der Talniederung leben können. Formen, die xerotherme Standorten bevorzugen (Pupilla triplicata, Truncatellina cylindrica), sind ausgesprochen selten.

Die Molluskenfauna der Enzschotter ist stark durch Zuwanderer aus dem südöstlichen Europa geprägt, die gegenwärtig in Südwestdeutschland nicht mehr vorkommen. Hierher gehören Taxa, welche inzwischen ausgestorben sind (Theodoxus serratiliniformis, Cochlostoma scalarinum saueri, Aegopis acieformis, Aegopis klemmi, Zonitoides sepultus) sowie ost- und südostalpine, nordbalkanische bzw. dinarische Faunenelemente wie Discus perspectivus, Aegopinella ressmanni, Macrogastra densestriata und Fusulus interruptus. Dazu kommen Formen, welche in Osteuropa und im östlichen Mitteleuropa ihr Verbreitungszentrum haben (Clausilia pumila, Perforatella bidentata und Pseudotrichia rubiginosa). Viel seltener sind Zuwanderer aus West- und Südwesteuropa (Azeca goodalli und Belgrandia germanica). Das gemeinsame Auftreten dieser Formen lässt auf klimatische Verhältnisse schließen, die sich von den heutigen wesentlich unterschieden haben. Das Klima dürfte durchschnittlich wärmer und feuchter und außerdem ausgeglichener gewesen sein.

Bei der Beurteilung des Alters einer Fauna ist die Anwesenheit von Arten von Interesse, die nur in ganz bestimmten Abschnitten des Pleistozäns auftreten. Einigen dieser Arten kommt darüber hinaus besondere Bedeutung zu, weil sie für bestimmte quartäre Warmzeiten geradezu kennzeichnend zu sein scheinen und oft in ganz charakteristischer Weise miteinander vergesellschaftet sind (LOŽEK 1986 \& 1997, HorÁČEK \& LOŽEK 1988). Folgende Taxa spielen in diesem Zusammenhang eine entscheidende Rolle (Tabelle 2): 
Tab. 2: Biostratigraphisch bedeutsame Molluskenarten aus den Enzschottern von Bietigheim-Bissingen und anderen, verwandten Faunen aus dem älteren Pleistozän Süd- und Mitteldeutschlands

Tab. 2: Biostradigraphically important mollusc species found in the gravels of the river Enz at BietigheimBissingen, and in related Pleistocene faunas of Middle and South Germany.

1) Enzschotter von Bietigheim-Bissingen, 20-22,5 m über Enzniveau

(Geyer 1914, Bibus \& RÄhle 2003).

2) Mittelpleistozäne Kalktuffe von Schmiechen bei Blaubeuren

(Dehm 1951, Lozek 1964a,b).

3) Mittelpleistozäne Spaltenfüllung am Adlerberg bei Nördlingen

(Dенм 1969) - veröffentlicht wurden nur die hier verzeichneten Arten.

4) Weißenburg 7, Spaltenfüllung im Steinbruch der Marmorwerke Weißenburg,

Fränkische Alb, mit altbiharischer Säugetierfauna (Dенм 1971).

5) Breitenberghöhle bei Gößweinstein, Fränkische Schweiz (HüssLEıı 1957, 1958), vermischte Höhlensedimente mit Resten von alt- und mittelpleistozänen Säugetieren.

6) Mittelpleistozäne Sand- und Tuffablagerungen von Brüheim bei Gotha (Hocker1898, 1907, MANıム 1973, 1983)

7) Bilzingsleben I (Thüringen), Theodoxus-Horizont (MANı 1983, Tab.1, Spalten 20-25 und Tab.4, Spalte 4); M^Nıı 1995, 2002), älteres Mittelpleistozän.

\begin{tabular}{|c|c|c|c|c|c|c|c|}
\hline & 1 & 2 & 3 & 4 & 5 & 6 & 7 \\
\hline Aegopis klemmi & $\mathrm{x}$ & - & & $\mathrm{x}$ & - & - & - \\
\hline Aegopies verticillus / acieformis & $\mathrm{x}^{1)}$ & $\mathrm{x}^{1)}$ & & $x^{1)}$ & $\mathrm{x}$ & $\mathrm{x}^{2)}$ & $\mathrm{x}$ \\
\hline Azeca goodalli & $\mathrm{x}$ & $\mathrm{x}$ & $\mathrm{x}$ & $\mathrm{x}$ & $\mathrm{x}$ & $\mathrm{x}$ & $\mathrm{x}$ \\
\hline Belgrandia germanica & $\mathrm{x}$ & $\mathrm{x}$ & & - & - & - & $\mathrm{x}$ \\
\hline Clausilia rugosa parvula & $\mathrm{x}$ & $\mathrm{x}$ & & $\mathrm{x}$ & $\mathrm{x}$ & $\mathrm{x}$ & $\mathrm{x}$ \\
\hline Cochlostoma scalarinum saueri & $\mathrm{x}$ & $\mathrm{x}$ & & $\mathrm{x}$ & - & - & - \\
\hline Fusulus interruptus & $\mathrm{x}$ & $\mathrm{x}$ & $\mathrm{x}$ & $\mathrm{x}$ & $\mathrm{x}$ & $\mathrm{x}$ & - \\
\hline Macrogastra densestriata & $\mathrm{x}$ & - & & $\mathrm{x}$ & $\mathrm{x}$ & $\mathrm{x}$ & - \\
\hline Platyla similis $(=$ diluviana $)$ & - & $\mathrm{x}$ & $\mathrm{x}$ & - & - & $\mathrm{x}$ & - \\
\hline Ruthenica filograna & - & $\mathrm{x}$ & & - & $\mathrm{x}$ & $\mathrm{x}$ & $\mathrm{x}$ \\
\hline Theodoxus serratiliniformis & $\mathrm{x}$ & - & & - & - & - & $\mathrm{x}$ \\
\hline Zonitoides sepultus & $x ?$ & $\mathrm{x}$ & $\mathrm{x}$ & - & - & - & - \\
\hline
\end{tabular}

1) Den Schalenmerkmalen nach zu acieformis gehörend.

2) Bei HOCKER (1907) als "Zonites acieformis" und bei MANIA (1973, Tab.17) als

"Aegopis verticillus" aufgeführt. 
Theodoxus serratiliniformis (GEYER 1914)

In den Enzschottern entfällt ungefähr 1/8 aller in den Sedimentproben vorhandenen Schalen auf diese Schnecke. Th. serratiliniformis wird von Meijer (1988 \& 1989) als jüngeres Synonym von Th. danubialis angesehen. MANIA (1995, 2002) sowie GlöER (2002) teilen diese Ansicht nicht und halten serratiliniformis für eine mit danubialis zwar nahe verwandte aber eigenständige, im Verlaufe des Mittelpleistozäns ausgestorbene Form. In Württemberg lassen sich Funde in den „Älteren Fluviatilen Schichten" von Steinheim a.d. Murr (FALKner 1984) zu den Vorkommen in den Enzschottern von Bietigheim-Bissingen in Beziehung setzen. In Bilzingsleben bei Sömmerda (Thüringen) ist Th. serratiliniformis Leitfossil der so genannten "Theodoxus-Schotter", welche von ManiA (1995 \& 2002) nach eingehender Analyse der Terrassenfolge in die fünftletzte Warmzeit gestellt werden (Bilzingsleben I, OIS 13). Andere, bei Mania (1973) verzeichnete Funde im Elbe-Saale-Gebiet sowie die Funde von Th. serratiliniformis in der Paludinenbank von Berlin (Schmierer 1923) stammen möglicherweise aus der selben Zeit.

\section{Cochlostoma scalarinum saueri (GEYER 1914)}

Die Art Cochlostoma scalarinum (VILLA 1841) lebt heute in den Küstengebieten der östlichen Adria von Istrien bis Griechenland. Bei der Subspezies saueri, für welche die Enzschotter von Bietigheim-Bissingen den klassischen Fundort darstellen, handelt es sich um eine ausgestorbene Form, die im älteren Mittelpleistozän im südlichen Mitteleuropa beheimatet war. $\mathrm{Ob}$ es sich dabei tatsächlich um eine Unterart von scalarinum handelt, ist keineswegs sicher. C. scalarinum saueri ist bisher nur von wenigen Plätzen bekannt. In Süddeutschland wird sie außer von Bietigheim-Bissingen aus alten Kalktuffen im Riether Tal bei Enzweihingen (Kranz \& Geyer 1922) sowie aus den Kalktuffen von Schmiechen bei Blaubeuren erwähnt (Dенм 1951). Dazu kommen Nachweise in einer Spaltenfüllung von Weißenburg (Fränkische Alb), die nach der begleitenden Säugerfauna als cromerzeitlich eingestuft wurde (Dенм 1971, von Koenigswald 1971). Auch aus der westlichen und östlichen Slowakei (alt- bis mittelpleistozäne Travertine von Hradišstě pod Vrátnom, Bojnice Úboče und Muráň-Piecky) liegen Funde vor (LožEK 1964b). Was die ökologischen Ansprüche der Schnecke angeht, so ist sie nach unserer Ansicht nicht zu den Offenlandarten (LožEK 1964b: 156), sondern zu den schattenliebenden Arten mesophiler Prägung zu stellen (Tabelle 1).

\section{Fusulus interruptus (C. PFEIfFER 1828)}

Diese suidostalpine Waldart war bisher aus den Enzschottern von Bietigheim-Bissingen noch nicht bekannt. In den niederösterreichischen und steirischen Kalkalpen und im Grazer Bergland hat Fusulus interruptus ein geschlossenes Areal besetzt und ist dort von der collinen bis in die montane Stufe zu finden, u.a. auch in Auwäldern (Frank 1975a, b). Im Mittelpleistozän ist die Art bis nach Mitteldeutschland vorgestoßen. Es ist denkbar, dass es sich dabei um ein einmaliges Ereignis in einer bestimmten Warmzeit gehandelt hat, denn die aus Deutschland überlieferten Faunen mit Fusulus interruptus sind, was ihre Zusammensetzung betrifft (vgl. auch Tabelle 2), einander sehr ähnlich.

Die Schnecke ist in Deutschland aus den mittelpleistozänen Kalktuffen von Schmiechen bei Blaubeuren am Südrand der Schwäbischen Alb (Dенм 1951), aus einer mittelpleistozänen Spaltenfüllung am Adlerberg bei Nördlingen (Dенм 1969) und aus der Spaltenfülllung Weißenburg 7 mit altbiharischen Säugern im Fränkischen Jura (Dенм 1971) bekannt. Dazu kommen Funde in der Breitenberghöhle bei Gößweinstein in Oberfranken (HÄssLein 1957, 1958), zusammen mit alt- bis jungbiharischen Wirbeltieren (BRUNNER 1957), und schließlich Funde aus mittelpleistozänen Kalktuffen von Brüheim bei Gotha in Thüringen (Hocker 1898, 1907). 
Clausilia rugosa parvula A. FÉrussac 1807

Im Jahre 1990 hat NorDsieck festgestellt, dass die gegenwärtig in Mitteleuropa heimische Rasse von Clausilia rugosa, Cl. rugosa parvula, in altpleistozänen Fundzusammenhängen und solchen aus dem älteren Mittelpleistozän nicht vorkommt und von einer Form vertreten wird, die sich von parvula gehäusemorphologisch beträchtlich unterscheidet. Sie wurde von Nordsieck (1990) als Cl. rugosa antiquitatis neu beschrieben. Wann genau und vor allem wie sich der Austausch der beiden Formen abgespielt hat, ob die beiden Formen eine Zeit lang nebeneinander existiert haben oder ob sie sich gegenseitig ausschließen, ist noch weitgehend ungeklärt.

Es ist deshalb von besonderem Interesse, dass aus den Enzschottern von Bietigheim-Bissingen ganz eindeutig Cl. rugosa parvula vorliegt, während in der nur $5 \mathrm{~km}$ entfernt liegenden Ziegelei Nestrasil in Besigheim in Lössderivaten, die mindestens der fünft- und sechstletzten Kaltzeit zuzuordnen sind und hochkaltzeitliche Faunen führen, ausschließlich $\mathrm{Cl}$. rugosa antiquitatis nachzuweisen ist (RÄHLE 1989). In diesem Zusammenhang ist anzumerken, dass GeYer (1914) in den Enzschottern eine Clausilienart gefunden hat, welche er nur mit großem Vorbehalt zu Clausilia bidentata stellte. Es ist nicht auszuschließen, dass es sich dabei um ein Exemplar der zum Verwechseln ähnlichen $\mathrm{Cl}$. rugosa antiquitatis gehandelt hat.

\section{Aegopis klemmi Schlickum \& LOZEK 1965}

Schalen dieser nach Fundstücken aus der Hundsheimer Höhle bei Bad Deutsch-Altenburg in Niederösterreich (Locus typicus), aus der Zlatý Kůn̆-Höhle bei Koněprusy (Böhmen), der Skalka Höhle bei Nové Mesto nad Vahom (Slowakei) und aus dem Travertin von Monosbel am Westfuß des Bükk-Gebirges (Ungarn) beschriebenen Art sind schon Geyer aufgefallen. Sie sind in seiner Artenliste (Geyer 1914: 126) als „Zonites sp. ?" aufgeführt. Ein entsprechendes Belegstück findet sich noch in der GEYERschen Sammlung im Staatlichen Museum für
Naturkunde in Stuttgart. Jetzt liegt ein weiteres Exemplar aus den Enzschottern vor. Es hat einen Durchmesser von $13,5 \mathrm{~mm}$, ist also noch nicht ganz erwachsen.

Bisher ist Aegopis klemmi in Deutschland erst ein Mal gefunden worden und zwar, wie in den Enzschottern mit Cochlostoma scalarinum saueri und Fusulus interruptus vergesellschaftet, in der cromerzeitlichen Spaltenfüllung Weißenburg 7 (s.o.). Die meisten Funde stammen jedoch aus dem östlichen Mitteleuropa, wo Ae. klemmi als Leitart für das ältere Mittelpleistozän (Hundsheimer Spalte) und das jüngere Altpleistozän bezeichnet werden kann (HoRÁČ́E \& LOŽEK 1988). Die Art scheint am Beginn des jüngeren Mittelpleistozäns ausgestorben zu sein.

\section{Zonitoides sepultus LOzEK 1964}

Es handelt sich um eine nach HorÁč́K \& LOžEK (1988) am Ende des älteren Mittelpleistozäns ausgestorbene Art, die an Waldfaunen gebunden gewesen zu sein scheint. Sie wurde in Böhmen (Zlatý Kůñ bei Koněprusy) und in Mähren (Stranská skala bei Brünn) in Sedimenten gefunden, die als "cromerzeitlich" eingestuft wurden (LOŽ̃K 1964a). In der WestSlowakei und in Ungarn ist Zonitoides sepultus in verschiedenen mittelpleistozänen Travertinen nachgewiesen (LOŽEK 1964a) und tritt dort zusammen mit Cochlostoma scalarinum saueri auf. In Deutschland war die Art bisher nur aus den Kalktuffen von Schmiechen bei Blaubeuren (Dенм 1951, Ložek 1964a, b) und aus der mittelpleistozänen Spaltenfüllung vom Adlerberg bei Nördlingen bekannt (Dенм 1969). Aus den Enzschottern liegt jetzt ein einzelnes Gehäuse vor, dessen Bestimmung aufgrund stärkerer Beschädigung nicht ganz sicher ist.

Die mit den Molluskenbeständen der Enzschotter nahe verwandten Faunen von Schmiechen, Adlerberg und Brüheim (Tabelle 2) teilen außerdem das Vorkommen von Platyla similis (Reinhardt 1880) (= diluviana Hocker 1907), einer süd- und südosteuropäischen Art, welche in Interglazialen des jüngeren Alt- und älteren 
Mittelpleistozäns zuweilen weit nach Nordwesten vorgestoßen ist und sogar England erreicht hat (Boeters et al. 1989).

Unter Berücksichtigung aller chronostratigraphisch verwertbarer Daten darf angenommen werden, dass die vorliegende Fauna aus den Enzschottern aus einer Warmzeit des früheren Mittelpleistozäns stammt. Darunter verstehen wir bezüglich der Interglaziale den Abschnitt zwischen Cromer II und Cromer IV i.S.v. ZAGwijN (1989). Geht man davon aus, dass Fusulus interruptus im Mittelpleistozän nur ein einziges Mal nach Nordwesten vorgestoßen ist, wie das von LOŽEK (1997) vermutet wird, und Faunen mit Fusulus interruptus gleiches Alter haben, dann ist, aufgrund des Auftretens altbiharischer Kleinsäuger in der Spaltenfüllung Weißenburg 7, für die Molluskenfauna aus den Enzschottern von Bietigheim-Bissingen ein prämindel- bzw. cromerzeitliches Alter anzunehmen. Ein solches Alter lässt sich mit der bisherigen Kenntnis der Enz- und Neckarterrassen und deren Deckschichten (Bibus \& Wesler 1995) recht gut vereinbaren. Danach gehören die Enzschotter von Bietigheim-Bissingen zu einem Komplex verschiedener, fast gleich hoher Terrassen zwischen $19-25$ m über der Enz, dessen älteste Anteile (aufgeschlossen in der Ziegelei Nestrasil in Besigheim, Bibus 1989a, 2002) mindestens in die siebtletzte und dessen jüngste Anteile mindestens in die fünftletzte Kaltzeit gehören. Die molluskenführenden Schotter scheinen demnach in der fünft- oder sechstletzten Warmzeit abgelagert worden sein.

Bibus (2002) hat die Schotter in Bietigheim-Bissingen aufgrund der terrassenmorphologischen Situation mit einem gewissen Vorbehalt in die fünftletzte Warmzeit und damit in das Cromer IV (OIS 13) gestellt. Aufgrund des gemeinsamen Vorkommens von Theodoxus serratiliniformis ist außerdem eine Parallelisierung mit dem Theodoxus-Horizont von Bilzingsleben möglich, den Mania $(1995,2002)$ ebenfalls der fünftletzten Warmzeit zuordnet.

Berücksichtigt man alle bisher vorliegenden
Daten, dann erscheint es am wahrscheinlichsten, dass wir es bei den molluskenführenden Mittelterrassenschottern der Enz von Bietigheim-Bissingen mit Sedimenten zu tun haben, die in der fünftletzten Warmzeit zur Ablagerung kamen. Eine derartige Altersbestimmung, die sich sowohl an der Terrassenabfolge als auch am Fossilbericht orientiert, lässt weiter reichende Schlussfolgerungen bezüglich des Alters anderer süd- und mitteldeutscher Fossillagerstätten zu, die Molluskenfaunen mit Fusulus interruptus führen aber nicht mit Flussterrassen in Verbindung gebracht werden können, wie die in Tabelle 2 verzeichneten Kalktuffe (Brüheim bei Gotha, Schmiechen bei Blaubeuren), Spaltenfüllungen (Weißenburg 7, Adlerberg bei Nördlingen) und Höhlensedimente (Breitenberghöhle bei Gößweinstein).

\section{Die eemzeitlichen \\ Terrassensedimente im Bereich des Heilbronner Beckens}

Aus einer Straßenbaustelle am sw-lichen Ortsrand von Heilbronn-Böckingen wurde von Bibus \& RÄhte: (1986) eine mit Oberkante ca. $5 \mathrm{~m}$ über der Talaue liegende Terrasse beschrieben, die mit einem eemzeitlichen, schneckenführenden Hochflutlehm abschloss. In den Lößdeckschichten konnten typische Paläoböden der Würmkaltzeit mit einer basalen Mosbacher Humuszone nachgewiesen werden. In vergleichbarer Reliefposition waren nunmehr im Heilbronner Becken drei weitere Terrassenkörper aufgeschlossen, in deren abschließenden Sanden, Kiesen und Hochflutlehmen Schnecken nachgewiesen werden konnten. Bei den Schotterkörpern handelt es sich nach der Höhenlage um die als rißzeitlich (vorletztkaltzeitlich) angesehene 5-7 m-Terrasse (Schotteroberkante) i.S.v. Bibus (2002: 115ff.) bzw. um die 12. Terrasse von Achildes (1939: 59) mit Felsbasis in $3 \mathrm{~m}$ über Talaue, die von AchiLLES (1939) allerdings nach unserer Auffassung 
fälschlich in die Würmkaltzeit gestellt wird. Der erste Aufschluss lag ca. 500 m westlich von der oben erwähnten Baustelle von Böckingen in der Ludwigsburger Straße 242-244 im untersten Talhangbereich unmittelbar über der Talaue. Es trat an der Basis ein in eine flache Rinne abtauchender sandig-lehmiger Kies in einer maximalen Mächtigkeit von 1,50 m auf (vgl. Abb. 3). Er war im oberen Bereich von einem grauen Gr- und im unteren Bereich von einem rostfarbenen Go-Horizont überprägt. Nahe der Terrassenkante lag der Kies auf grünblauen, feinsandigen Tonsteinen in der flachen Rinne auf einer harten Dolomitbank (Unter- keuper?). Die Terrassenoberkante konnte in ca. $5 \mathrm{~m}$ über der Talaue eingemessen werden. Die Schotter wiesen nach einer durchgeführten Schotteranalyse mit ca. $90 \%$ einen sehr hohen Anteil an Kalkgeröllen auf, wobei der Jurakalk mit $65 \%$ im Grobkies und 72,5\% im Mittelkies gegenüber dem Muschelkalk mit $23 \%$ im Grobkies und $18 \%$ im Mittelkies dominierte. Der Rest wurde von Buntsandsteinen (Grobkies 3,5\%, Mittelkies 1,5\%), Keupersandsteinen (Grobkies 3,5\%, Mittelkies $4 \%$ ) sowie Quarzen, Quarziten, verkieselten Gesteinen sowie Schluff- und Tonsteinen des Keupers gebildet, die jeweils 1,5\% nicht überstiegen.
W
$E \mid N$

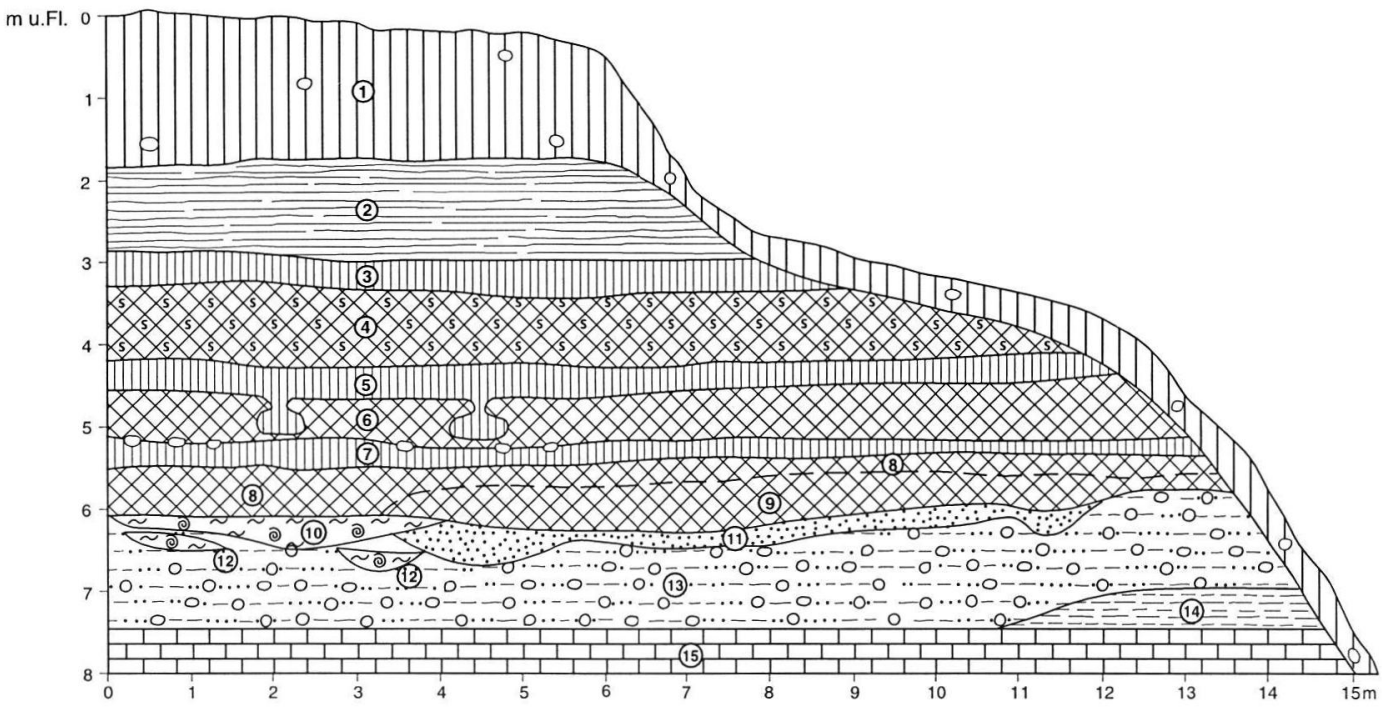
(1) Hangkolluvium, M
(2) Schwemmsediment, feingeschichtet
(3) fahlgrauer Ah-Horizont
(4) Hochflutlehm mit GoBvt-Horizont
(5) diffuser Ah-Horizont
(6) Hochflutlehm mit $\mathrm{B}_{\mathrm{t}}$-Horizont
(7) diffuser $\mathrm{Ah}$-Horizont
(8) Hochflutlehm mit Bt-Horizont

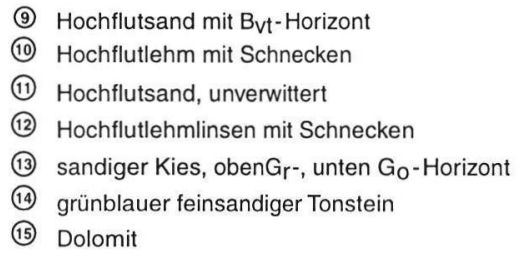

Abb. 3: Aufschlussprofil im untersten Hangbereich in der Baustelle Ludwigsburger Straße 242-244 in Heilbronn-Böckingen

Fig. 3: Outcrop section at the lowermost part of the slope within the construction site Ludwigsburger Straße 242-244 at Heilbronn-Böckingen. 
Der Kies wurde von unverwitterten Sanden überlagert, die in kleinen Rinnen in den liegenden Kies eingriffen (Abb. 3, Schicht 11). In der W-Ecke des Aufschlusses wurden die Hochflutsande wiederum von Rinnen geschnitten, in denen rostbraun-grau marmorierte, schneckenführende Hochflutlehme zur Ablagerung gelangt waren. Aus diesem Bereich konnten Schnecken ausgelesen werden. Darüber folgte ein $3 \mathrm{~m}$ mächtiger Komplex von kalkfreien braunen Lehmen und Tonen, welche ebenfalls als Hochflutlehme gedeutet werden. Die Abfolge wurde von zwei humosen Zonen (Nr. 5, 7) gegliedert und von einer dritten abgeschlossen (Nr. 3). Die drei unter den humosen Zonen liegenden Hochflutlehme waren kalkfrei und wiesen Tonüberzüge auf den Aggregatflächen auf, so dass es sich zweifelsfrei um Bt-Horizonte handelte (Nr. 4, 6, 8). Der unterste Bt-Horizont griff mit einem Bvt-Horizont auf die liegenden Hochflutsande und -lehme über, was für eine in situ-Bildung spricht. Zudem wies er an seiner Obergrenze eine durch Gerölle angezeigte Diskordanz auf. Der oberste Bt-Horizont besaß an seiner Untergrenze einige Taschen, mit denen er den liegenden Ah-Horizont überprägt hatte. Auch diese Erscheinung ist als Zeichen einer in situ-Bodenbildung zu werten. Von den drei AhHorizonten sind die beiden unteren verbraunt und schwach entwickelt. Der oberste Ah-Horizont besaß hingegen eine kräftige Ausbildung. Durch hydromorphe Einflüsse wies er eine fahldunkelgraue Färbung, große Rostflecken und rostimprägnierte ehemalige Wurzelröhren auf. Eine durchgeführte Pollenanalyse hat leider keine auswertbaren Ergebnisse erbracht (KNIPPING, schriftl. Mitt.).

Auf dem pedogen überprägten Hochflutlehmkomplex lag ein feingeschichtetes, kalkhaltiges Abschwemmsediment aus millimeterfeinen, horizontalen Schluff- und schluffigen Lehmbändern, bei dessen Ausgangssubstrat es sich um Löß und Humuszonenmaterial handeln dürfte (Schicht 2). Den oberen Profilabschluss bildete ein Kiese und Scherben führendes Kolluvium
(Schicht 1), welches hangabwärts sämtliche älteren Schichten kappte.

Bei den aus dem Hochflutlehm entnommenen Schnecken handelt es sich um das Fragment einer sehr feucht liebenden Molluskengesellschaft, wie sie in der Nähe von Fließgewässern in Hochstaudenfluren und Auwäldern anzutreffen ist (Tabelle 3, Spalte A). Das Auftreten von Aegopinella spec. und Ena montana weist die Faunula als warmzeitlich aus. Arten, die für irgendeine Warmzeit, z.B. auch für das Eem, spezifisch wären, sind nicht vorhanden. Die aquatische Komponente ist, anders als beispielsweise in den Hochflutsedimenten von Obereisesheim (Tabelle 3, Spalten B1 und B2), ausgesprochen spärlich vertreten.

Nach der geomorphologischen Position sollte es sich bei dem beschriebenen Terrassenkörper um die Rißterrasse mit dem abschließenden eemzeitlichen Hochflutlehm handeln. Allerdings treten am Talhang zwischen Böckingen und Klingenberg auch noch ältere Schotter (so genannte Hochterrasse, vgl. WIID 1968: 62 und GK 25 Bl. 6821 Heilbronn, Brunner 1986: 62f. und GK 25 Bl. 6821 Heilbronn) auf, deren Untergrenze durch synsedimentäre Absenkungen mit einem deutlichen Gefälle in das Heilbronner Becken einfällt. Die Basis dieser Schotter liegt jedoch am weslichen Ortsrand von Böckingen höher am Talhang. Andererseits kann es sich bei dem beschriebenen Vorkommen auch nicht um die ältere Niederterrasse aus dem beginnenden Mittelwürm handeln, da in den abschließenden kalkhaltigen Hochflutlehmen die interglazialen Schnecken gefunden wurden. Der Hochflutlehm muss deshalb, trotz fehlender letztinterglazialer Charakterarten der Eemwarmzeit zugeordnet werden. Interessant sind außerdem die durch Ah-Horizonte gegliederten und durch Bt-Horizonte überprägten Hochflutlehme. Wenn man den gesamten Komplex nicht in die Eemwarmzeit stellen will, ist er am ehesten mit dem altwürmzeitlichen Abschnitt der drei Mosbacher Humuszonen zu vergleichen. Es überrascht allerdings, dass auf den Hochflutlehmen 
Bt-Horizonte entwickelt sind und sich zwischen den Ah-Horizonten keine Al-Horizonte gebildet oder erhalten haben. Wenn allerdings das Ausgangsmaterial sehr stark vorverwittert ist, was bei Hochflutlehmen der Fall sein kann, kommt es u.U. sehr schnell zu einer Tonwanderung und zu Toncutanen. Selbst auf stark kalkhaltigem Löß sind an Stellen mit Wasserzuzug als Folge von Degradationserscheinungen Bt-Horizonte direkt unter Ah-Horizonten der Mosbacher Humuszonen beobachtet worden (vgl. SEMmEL 1996, 1999, Bibus et al. 1996). In diesen Fällen sind jedoch, im Gegensatz zum vorliegenden Profil, die Ah-Horizonte kräftiger und die BtHorizonte schwächer entwickelt. Außerdem wurde von Weidenfelier et al. (1999: 26) gezeigt, dass es im Profil Koblenz-Metternich, übrigens in ähnlicher Unterhangposition wie in Böckingen, im Altwürm zur Ausbildung von Bt-Horizonten gekommen ist, ein Sachverhalt, auf den bereits Rohdenburg \& Meyer (1966) und Rickren (1983) für die Lößgebiete in Niedersachsen hingewiesen haben. In besonderen Positionen der Lößlandschaft mit starkem lateralen Wasserzuzug sowie im feuchteren Randbereich des Lößgebietes scheint es somit in Altwürminterstadialen lokal zu einer Bt-Bildung gekommen zu sein. Der auf Hochflutlehmen entwickelte Bt-Horizontkomplex in Böckingen dürfte deshalb mit den Altwürminterstadialen parallelisiert werden. Zu dieser Deutung passt sehr gut, dass über der jüngsten Humuszone abluale Schwemmsedimente folgen, die in Lößaufschlüssen von Baden-Württemberg mehrfach in dieser Position gefunden und als Äquivalente der Niedereschbacher Zone in das frühe Mittelwürm gestellt wurden (vgl. BiBus 2002).

Im Industriegebiet von Obereisesheim, welches am W-Rand der Neckaraue liegt, war in einem weiteren Aufschluss (Silcherstraße 34) im untersten Bereich einer Terrassenstufe ein 3,85 m mächtiger Schotter mit Felsbasis in $151,4 \mathrm{~m}$ ü.NN aufgeschlossen. Die Obergrenze lag ca. 5 $\mathrm{m}$ über der Talaue im Niveau der rißzeitlichen 5-7 m-Terrasse i.S.v. Bibus (2002). Bei dem unteren Abschnitt der Kiese handelte es sich um schwach geschichtete, sandige Mittel- bis Grobkiese, die dachziegelartig abgelagert waren oder z.T. auch senkrecht standen. Darüber folgte mit einer deutlichen Grenze ein locker gelagerter Mittelkies, in dem im obersten Bereich sehr viele Schnecken eingelagert waren (vgl. Abb. 4 u. Tab. 3, Spalte B1). Dieser Kies wurde von einem 1,80 m mächtigen, rostbraun-grau marmorierten, kalkhaltigen Hochflutlehm überlagert, der vor allem im oberen Abschnitt vereinzelt Schnecken führte (vgl. Tab. 3, Spalte B2). Abgeschlossen wurde der Terrassenkörper von einem grauen, sandigen Kiesband mit aufgearbeiteten Schneckenbruchstücken. Das Deckschichtenprofil setzte mit einer geringmächtigen Umlagerungszone aus einem schwach kiesigen, lehmigen Sand ein. Darüber folgte eine ca. 60 $\mathrm{cm}$ mächtige, intensiv ausgebildete Humuszone mit einzelnen braunen Degradationsflecken, die als Tigerfleckung bzw. als „Gefleckter Horizont" i.S.v. RoHDEnburg (1964) angesprochen werden können. Die Humuszone war im oberen Bereich durch weiche Kalkkonkretionen sekundär aufgekalkt. Der Ah-Horizont musste jedoch ursprünglich auch kalkhaltig gewesen sein, da in seinem unteren Abschnitt noch einige Schneckenbruchstücke gefunden werden konnten. Das Profil wurde von einem schwach verbraunten, schneckenführenden, feinsandigen Löß und dem Ap-Horizont abgeschlossen.

Obwohl das Deckschichtenprofil nicht stark gegliedert ist, kann aufgrund der erhaltenen altwürmzeitlichen Mosbacher Humuszone gefolgert werden, dass der untere Abschnitt der unterlagernden Terrasse in die Rißeiszeit (vorletzte Eiszeit) gehört und der obere Bereich mit den schneckenführenden, sandigen Kiesen und Hochflutlehmen in das Eem gestellt werden muss. Von Bedeutung ist in diesem Zusammenhang, dass ca. $1 \mathrm{~km}$ weiter nördlich die gleiche Terrasse beim Bau eines Rückhaltebeckens an der lößüberdeckten Terrassenstufe großräumig aufgeschlossen war (TK 25 Bl. 6721 Bad Friedrichshall; ca. R 351532 H 545150). 


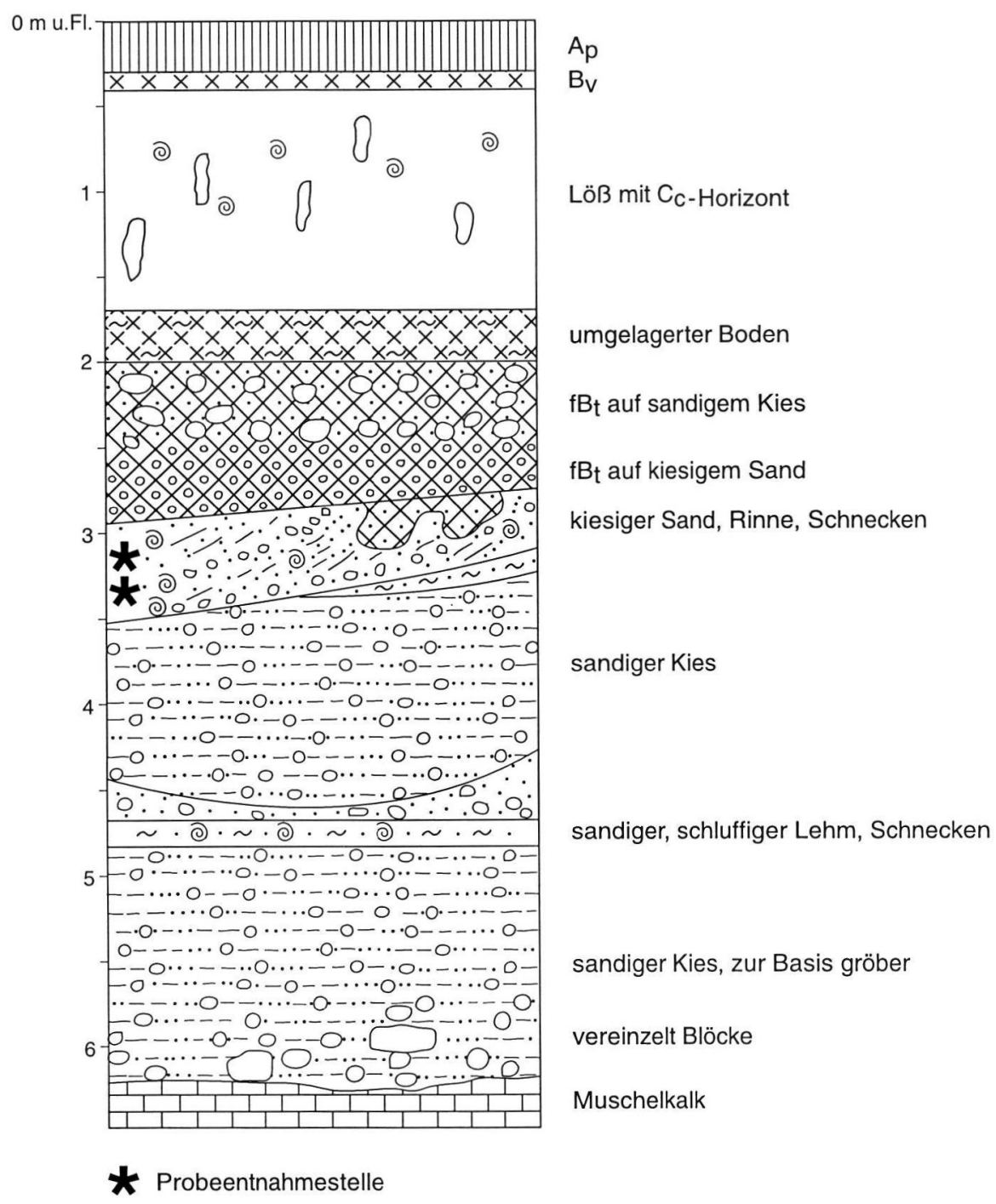

Abb. 4: Aufschlussprofil im Industriegebiet von Obereisenheim (Silcherstraße 34) im untersten Talhangbereich unmittelbar über der Talaue

Fig. 4: Outcrop section at the industrial area of Obereisesheim (Silcherstraße 34) at the lowermost part of the valley slope directly above the flood plain.

Auch hier wurde der Terrassenkörper von einem Hochflutlehm abgeschlossen, der nach unseren Untersuchungen die gleichen Schnecken wie im Industriegebiet von Obereisesheim führte. Unmittelbar über dem eemzeitlichen Hochflutlehm war an der Basis der Lößdeckschichten ebenfalls eine kräftige Mosbacher Humuszone ausgebildet. Darüber folgte noch Mittelwürmlöß mit der Niedereschbacher Zone, dem Lohner Boden und durch Nassböden gegliederter Jungwürmlöß. Bereits aufgrund der Deckschichten mit dem fast vollständigen Würmlöß muss der unterlagernde, schneckenführende Hochflutlehm auch an dieser Stelle zweifelsfrei in das Eem gestellt werden. 
Tab. 3: Mollusken aus eemzeitlichen Terrassensedimenten des Heilbronner Raumes. A: Heilbronn-Böckingen, Ludwigsburger Straße, Hochflutlehm; B1: Obereisesheim, Mittelkies; B2: Obereisesheim, Hochflutlehm.

\section{Angegeben sind die Dominanzwerte}

(prozentuale Anteile der einzelnen Arten an der Gesamtindividuenzahl) in \%.

!! - hochwarmzeitliche Indexarten, ! - warmzeitliche Arten, (!) - vorwiegend warmzeitliche Arten.

Tab. 3: Molluscs from Eemian terrasse-sediments in the area of Heilbronn. A. Heilbronn-Böckingen, Ludwigsburgerstraße, high-flood loam ; B1: Obereisesheim, gravels; B2: Oberheisesheim, high-flood loam

The frequency of species per sample is expressed by dominance rates in \%.

!! - index species of interglacials, ! - species characteristic of warm phases, (!) - Mainly in warm phases, but also in warmer periods of glacials.

A $\quad$ B1 B2

1. Ausgesprochene Waldarten

! Aegopinella spec. (große Art)

! Cochlodina laminata

! Ena montana

!. Monachoides incarnatus

2. Andere Schatten liebende Arten

Arianta arbustorum $\quad 28,6$

Clausilia pumila $\quad 14,3$

Eucobresia diaphana

(!) Fruticicola fruticum

Vitrea crystallina

Trichia striolata

$\begin{array}{ccc}4,1 & 0,2 & - \\ - & 0,1 & - \\ 4,1 & - & - \\ - & 0,1 & -\end{array}$

3. Ubiquisten

Agriolimacidae / Limacidae

Carychium tridentatum

Cochlicopa lubrica

Punctum pygmaeum

Succinella oblonga

Trichia hispida

\section{8,6
14,3}

2,0

12,3

16,3

B1 $\mathrm{B} 2$

,

$0,3 \quad 1,8$

$0,6 \quad-$

$0,3 \quad 3,5$

$\begin{array}{ccc}- & 1,6 & 38.5 \\ - & 0,2 & - \\ 16,3 & 1,5 & - \\ - & 0.8 & - \\ - & 0,2 & - \\ - & 1,8 & 8,8\end{array}$

$\begin{array}{cc}0,3 & 3,5 \\ - & -\end{array}$
4. Offenlandarten

Chondrula tridens

Pupilla muscorum

Vallonia costata

Vallonia excentrica

Vallonia pulchella

(!) Vertigo pygmaea

5. Wasser- und Sumpfmollusken

(!) Ancylus fluviatilis Anisus leucostoma

!! Belgrandia germanica

(!) Bithynia tentaculata

Galba truncatula

Gyraulus crista

Pisidium nitidum

Planorbis spec.

Radix ovata

Succineidae (große Arten)

! Theodoxus fluviatilis

Valvata cristata

Valvata piscinalis

(!) Vertigo antivertigo

Artenzahl:

Individuenzahl:
A

B1 B2

$\begin{array}{ccc}- & 0,2 & - \\ - & 0,2 & - \\ - & 0,8 & 8.8 \\ - & - & 1,8 \\ - & 11,5 & - \\ - & 1,3 & 1,8\end{array}$


Die Fauna setzt sich überwiegend aus aquatischen Arten und feuchtliebenden terrestrischen Arten der Talaue zusammen. Das ökologische Spektrum reicht von Bewohnern fließenden Wassers (Theodoxus, Belgrandia, Radix ovata, Ancylus fluviatilis) bis zu Arten temporärer Kleingewässer und Sümpfe (Valvata cristata, Galba truncatula, Vertigo antivertigo und große Succineidae). In den terrestrischen Arten spiegelt sich eine weitgehend offene Landschaft wider, denn Arten, die auf höhere Vegetation angewiesen sind, spielen nur eine ganz untergeordnete Rolle. Unter den Offenlandarten ist Vallonia pulchella, eine Art, die sich mit Vorliebe auf feuchten Wiesen aufhält, besonders häufig. Aus dem Hochflutlehm (Tabelle 3, Spalte B2) liegen vergleichsweise wenige Fossilien vor. Die Fauna erscheint insgesamt klimatisch viel anspruchsloser als diejenige aus dem Liegenden. Warmzeitliche Elemente fehlen fast völlig. Von dem festschaligen Theodoxus fluviatilis waren nur noch Fragmente zu finden. Es ist zu vermuten, dass es sich dabei um umgelagertes Schalen handelt und dass die Art zur betreffenden Zeit schon nicht mehr im Neckar gelebt hat. Wie im Falle von Heilbronn-Böckingen (BıBus \& RÄHLE 1986) zeichnet sich auch in der Fauna von Obereisesheim von unten nach oben eine Klimaverschlechterung ab.

Bemerkenswerterweise wurde von НБuвaCH (1925: 55f.) nur $1,5 \mathrm{~km}$ weiter nördlich angenommen, dass gegenüber der Kochermündung eine beachtliche jungquartäre Tektonik geherrscht hat, durch welche die würmzeitliche Niederterrasse um über $5 \mathrm{~m}$ herausgehoben sein soll. Diese Ansicht wurde auch in den Erläuterungen der GK Bl. $6721 \mathrm{Bl}$. Bad Friedrichshall (BRUnNer 2001: 72, Beil. 5c) vertreten, wobei die Schotteroberkante des als herausgehoben angesehenen Würmschotters bis $160 \mathrm{~m}$ ü.NN und damit sogar ca. $12 \mathrm{~m}$ über der Aue liegen soll. Aufgrund der tiefen Lage der Rißterrasse und der eemzeitlichen Sedimente nördlich von Obereisesheim und im übrigen Heilbronner Becken (BiBus 2002) gehen wir davon aus, dass gegenüber der Kochermündung keine gehobene Niederterrasse, sondern eine ältere mittelpleistozäne Terrasse vorliegt, zumal der Schotterkörper auch mit mächtigen Lößdeckschichten überkleidet wurde. Bereits KoKEn (1900) hat die Schotter gegenüber der Kochermündung zu seiner präwürmzeitlichen, jüngeren Hochterrasse gestellt (vgl. auch Einstufung auf der GK Bl. 6721 Bad Friedrichshall). Die Vorstellung einer intensiven tektonischen Heraushebung nach der Niederterrassenaufschüttung von 5 bis u.U. sogar über $10 \mathrm{~m}$ halten wir deshalb, auch aufgrund der Molluskenfunde, für nicht zutreffend.

\section{Schriftenverzeichnis}

Achilles, K. A. (1939): Diluvialgeologische Untersuchungen im mittleren Neckartal. Jh. Ver. vaterl. Naturkde. Württ., 95: 1-110, 10 Phot., 6 Abb., 26 Tab., 4 Taf.; Stuttgart.

Bibus, E. (1989a), mit Beiträgen von W. RÄHLE \& L. ZölLER: Programm und Exkursionsführer zur 8. Tagung des Arbeitskreises „Paläoböden" der Deutschen Bodenkundlichen Gesellschaft, 25.5.-27.5.1989 in Heilbronn. - 31 S., 18 Abb.; Tübingen.

Bibus, E. (1989b): Zur Gliederung, Ausbildung und stratigraphischen Stellung von Enzterrassen in Großbaustellen bei Vaihingen an der Enz. - Jh. geol. L.-Amt Baden-Württ., 31: 7-22, 5 Abb.; Freiburg i. Br.

Bibus, E. (2002): Zum Quartär im mittleren Neckarraum - Reliefentwicklung, Löß/Paläobodensequenzen, Paläoklima. - Tübinger geowiss. Arb., D8: 236 S., 45 Abb., 2 Tab., 8 Phot.; Tübingen.

Bibus, E., Bludau, W., Bross, C. \& Rähle, W. (1996): Der Altwürm- und Rißabschnitt im Profil Mainz-Weisenau (Heidelberger Zement AG) und die Eigenschaften der Mosbacher Humuszone. - Frankfurter geowiss. Arb., D, 20: 21-52, 5 Abb., 2 Tab.; Frankfurt a.M. 
Bibus, E. \& RÄHLE, W. (1986): Geomorphologische Lage, Deckschichten und Molluskenführung letztinterglazialer Hochflutlehme im Neckartal (Baden-Württemberg). - Eiszeitalter u. Gegenwart, 36: 89-109, 7 Abb. 2 Tab.; Stuttgart.

Bibus, E. \& RÄHLE, W. (2003): Molluskenführung und Deckschichtengliederung von Höhenschottervorkommen bei Haigerloch (Eyachtal) und Rottenburg (Neckartal) in Württemberg. - 19 S., 4 Abb.; Tübingen (abgeschl. Manuskript).

Bibus, E. \& Westerr, J. (1995): The middle Neckar as an example of fluvio-morphological processes during the Middle and Late Quaternary period. - Z. Geomorph., N.F., Suppl.-Bd. 100: 15-26, 4 Abb.; Berlin-Stuttgart.

Boeters, H.D., Gittenberger, E. \& Subai, P. (1989): Die Aciculidae: (Mollusca: Gastropoda Prosobranchia). - Zool. Verhandelingen, 252: 1-234, 220 Abb.; Leiden.

Brunner, G. (1957): Die Breitenberghöhle bei Gößweinstein/Ofr..Eine Mindel-Riß und eine postglaziale Mediterran-Fauna. - N. Jb. Geol. Paläont. Mh., 1957: 352-403, 18 Abb.; Stuttgart.

Brunner, H. (1986): Erläuterungen zu Blatt 6812 Heilbronn. - GK 25 Baden-Württ.: 204 S., 6 Abb., 8 Tab., 1 Taf., 4 Beil.; Stuttgart.

Brunner, H. (2001): Erläuterungen zu Blatt 6721 Bad Friedrichshall. - GK 25 BadenWürtt.: 183 S., 24 Abb., 6 Tab., 7 Beil.; Freiburg i.Br.

Denм; R. (1951): Mitteldiluviale Kalktuffe und ihre Molluskenfauna bei Schmiechen nahe Blaubeuren (Schwäb.Alb). - N. Jb. Geol. Paläont. Abh., 93 (1951): 247-276, 2 Abb.,2 Taf.; Stuttgart.

Deнн, R. (1969): Über den Nachweis der mittelpleistozänen Warmzeit in Nordbayern. - Bayer. Akad. Wiss., math.-nat. K1., Sitzungsber. 1968: 12-15; München.

Dенм, R. (1971): Eine altpleistozäne Spalten- füllung von Weißenburg in Bayern und ihre Molluskenfauna. - Mitt. bayer. Staatssamml. Paläont. hist. Geol., 11: 77-85, Taf.8; München.

Falkner, G. (1984): Eine Molluskenfaunula aus den „Älteren Fluviatilen Schichten“ der Neuen Grube Sigrist von Steinheim a.d. Murr. - Documenta naturae, 18: 18-24, 1 Karte, 1 Tab., München.

Frank, C. (1975a): Molluskenassoziationen des Weizer Berglandes und der Fischbacher Alpen. - Mitt. dtsch. malakozool. Ges., 3: 212-231; Frankfurt a.M.

Frank, C. (1975b): Zur Biologie und Ökologie mittelsteirischer Landmollusken. - Mitt. naturwiss. Ver. Steiermark, 105: 225-263, 1 Abb., 17 Tab.; Graz.

Geyer, D. (1914): Über diluviale Schotter Schwabens und ihre Molluskenreste. - Jber. Mitt. oberrh. geol. Ver., N.F., 4: 120-138, 1 Taf.; Stuttgart.

GlöEr, P. (2002): Die Süßwassergastropoden Nord- und Mitteleuropas. Die Tierwelt Deutschlands, Teil. 73: 327 S., 307 Abb.; Hackenheim.

HÄsslein, L. (1957): Mollusken aus der Breitenberghöhle. In: G. BRunner: Die Breitenberghöhle bei Gößweinstein. - N. Jb. Geol. Paläontol. Mh. 1957: 388-393, Stuttgart.

HÄssicin, L. (1958): Bemerkenswerte Helicigona-Vorkommen im Diluvium einer fränkischen Höhle. - Arch. Moll., 87: 37-40, 6 Abb.; Frankfurt a.M.

Høubach, K. (1925): Beiträge zur Frage der Entstehung der Heilbronner Mulde. - Jh. Ver. vaterländ. Naturkde. Württ., 81: $37-$ 62, 2 Kt., 1 Taf.; Stuttgart.

Hocker, F. (1898): Die Conchylienfauna der diluvialen Sand- und Tuffablagerungen bei Brüheim im Herzogtum Gotha. - Nachrbl. dtsch. malakozool. Ges., 30: 86-91; Frankfurt a.M.

HoCkER, F. (1907): Nachtrag zum Verzeichnis der in der diluvialen Sand- und Tuffablagerung von Brüheim bei Gotha vorkommen- 
den Conchylien. - Nachrbl. dtsch. malakozool. Ges., 39: 86-93; Frankfurt a.M.

HoRÁČEK, I. \& LožEK, V. (1988): Palaeozoology and the Mid-European Quaternary past: scope of the approach and selected results. - Rozpravy Ceskoslovenske Akademie Ved, Rada matematickych a Prirodnich Ved, 98: 1-102 + 1-4, 14 Abb.+1, 16 Tab.+10+1, 7 Fotos; Praha.

JAECKEL, S. G. (1962): Ergänzungen und Berichtigungen zum rezenten und quartären Vorkommen der mitteleuropäischen Mollusken. In: P. Brohmer, P. Ehrmann \& G. Ulmer: Die Tierwelt Mitteleuropas, Bd. 2, Ergänzung (Lief.1): 25-260, 9 Taf.; Leipzig.

KoENIGSWALD, W. vON (1971): Die altpleistozäne Wirbeltierfaunula aus der Spaltenfüllung Weißenburg 7 (Bayern). - Mitt. bayer. Staatsamml. Paläont. hist. Geol., 11: 117-122, 3 Abb.; München.

KoKen, E. (1900): Begleitworte zur Geologischen Spezialkarte der Umgebung von Kochendorf 1: 10 000. - 79 S., 12 Kt., 1 Profiltaf.; Stuttgart (Lindemann).

Kranz, W. \& Geyer, D. (1922): Ein altes Hochtal im Gebiet des Grenzbach- und Strudelbachtals nördlich Weißach. - Jber. Mitt. oberrh. geol. Ver., N.F., 9: 66-77, 2 Abb.; Stuttgart.

LožEK, V. (1964a): Neue Mollusken aus dem Altpleistozän Mitteleuropas. - Arch. Moll., 93: 193-199, 6 Abb.; Frankfurt a.M.

Ložॅк, V. (1964b): Quartärmollusken der Tschechoslowakei. - Rozpravy Ustr. ust. geol., 31, 374 S., 91 Abb., 10 Tab., 32 Taf., 4 Beil., Praha.

LOŽCK, V. (1986): Quaternary Malacology and Fauna genesis in Central Europe. - Proc. 8th Intern. Malacolog. Congress, Budapest, 1983: 143-145; Budapest.

LožcK, V. (1997): Palaeoecology of Quaternary Molluscs. - Heldia, Sonderh. 5: 20-26; München.

Mania, D. (1973): Paläoökologie, Faunenentwicklung und Stratigraphie des Eiszeitalters im mittleren Elbe-Saalegebiet auf Grund von Molluskengesellschaften. - Geologie, 21, Beih. 78/79: 175 S., 14 Abb., 33 Tab.; Berlin.

Mania, D. (1983): Die Molluskenfauna des mittelpleistozänen Travertinkomplexes bei Bilzingsleben und ihre ökologisch-stratigraphische Aussage. - Veröff. Landesmus. Vorgesch. Halle, 36: 131-155, 3 Abb., 5 Tab.; Berlin.

MANiA, D. (1995): The influence of Quaternary climatic development on the Central European mollusc fauna. - Acta zool. cracov., 38 : 17-34, 15 Abb.; Krakow.

ManiA, D. (2002): Travertine und paläolithische Funde von Bilzingsleben, Landkreis Sömmerda. - Jber. Mitt. oberrh. geol. Ver., N.F., 84: 95-107, 9 Abb.; Stuttgart.

Meijer, T. (1988): Fossiele zoetwaternerieten uit het Nederlandse Kwartair en enkele opmerkingen over het voorkomen van deze groep in het Kwartair van Noordwest Europa. - De Kreukel, Jubileumnummer: 89109, 1 Taf., 4 Tab.; Amsterdam.

MEijER, T. (1989): Notes on quaternary freshwater mollusca of the Netherlands, with descriptions of some new species. - Meded. Werkgr. Tert. Kwart. Geol., 26(4): 145-181, 3 Tab. 2 Taf.; Leiden.

Nordsieck, H. (1990): Revision der Gattung Clausilia Draparnaud, besonders der Arten in SW-Europa (Das Clausilia rugosaProblem) (Gastropoda: Stylommatophora: Clausiliidae). - Arch. Moll., 119 (1988): 133-179, 11 Abb. 3 Taf.; Frankfurt a.M.

RÄHLE, W. (1989): Molluskenfaunen aus alt-, mittel- und jungpleistozänen Lössen der Umgebung von Heilbronn und Besigheim. - In: Bibus, E.: Programm und Exkursionsführer der 8. Tagung des Arbeitskreises "Paläoböden" der Deutschen Bodenkundlichen Gesellschaft (25.5. - 27.5.1989 in Heilbronn): 28-31; Tübingen.

RÄHLE, W. \& Bibus, E. (1992): Eine altpleistozäne Molluskenfauna in den Höhenschot- 
tern des Neckars bei Rottenburg, Württemberg. - Jh. geol. L.-Amt Baden-Württ., 34: 319-341, 3 Abb., 2 Tab.; Freiburg i. Br.

RickeN, W. (1983): Mittel- und jungpleistozäne Lößdecken im südwestlichen Harzvorland. Stratigraphie, fazielle Differenzierung und Konnektierung in Flussterrassen. - Catena, Supplem. 3: 95-138; Cremlingen.

Rohdenburg, H. (1964): Ein Beitrag zur Deutung des "Gefleckten Horizontes". - Eiszeitalter u. Gegenwart, 15: 66-71, 3 Abb.; Öhringen.

Rohdenburg, H. \& Meyer, B. (1966): Zur Feinstratigraphie und Paläopedologie des Jungpleistozäns nach Untersuchungen an südniedersächsischen und nordhessischen Lößprofilen. - Mitt. dtsch. bodenkdl. Ges., 5: 1-135; Göttingen.

Schlickum, W.R. \& Ložck, V. (1965): Aegopis klemmi, eine neue Interglazialart aus dem Altpleistozän Mitteleuropas.- Arch. Moll., 94 (3/4): 111-114, 3 Abb.; Frankfurt a.M.

Schmierer, T. (1923): Beitrag zur Kenntnis des faunistischen und floristischen Inhalts der Berliner Paludinenbank. - Z. dtsch. geol. Ges., 74: 207-236, 1 Taf.; Hannover.

Semmel, A. (1996): Stop 1, Paläoböden im

Würmlöß, insbesondere im Altwürmlöß des Steinbruchs Mainz-Weisenau - Problemstellung und Übersicht über die Forschungsergebnisse. - Frankfurter geowiss. Arb., Ser. D, 20: 11-20, 1 Abb., 1 Tab.; Frankfurt a.M.

Steusloff, U. (1953): Wanderungen und

Wandlungen der Süßwasser-Mollusken Mitteleuropas während des Pleistozäns. Arch. Hydrobiol., 48: 210-236, 4 Abb., 1 Tab.; Stuttgart.

Weidenfelder, M., Boenigk, W., Frechen, M., Hambach, U. \& Reinders, J. (1999): The late Middle and Upper Pleistocene loess/paleosol deposits of section KoblenzMetternich, Mosel valley. - In: WeIDEnfELLER, M. \& Zöller, L. [eds.]: Loess in the Middle and Upper Rhine Area, Field Guide. - Geol. L.-Amt Rheinland-Pfalz: 17-30, 8
Abb.; Mainz.

WitD, H. (1968): mit Beiträgen von O. LiNCK et al.: Erläuterungen zu Blatt 6821 Heilbronn. - GK 25 Baden-Württemberg. - 183 S., 11 Abb., 2 Taf.; Stuttgart.

ZaGiwin, W.H. (1989): The Netherlands during the Tertiary and the Quaternary: A case history of coastal lowland evolution. - Geologie en Mijnbouw, 68: 107-120, 23 Abb.; Dordrecht. 